\title{
Quality of working life and social support with the mediating role of resiliency
}

\author{
Maryam Amini $^{{ }^{a *}}$, Mahbobeh Chinaveh ${ }^{\mathrm{b}}$ and Fereshteh Mostafavi rad $^{\mathrm{b}}$
}

${ }^{a}$ Department of psychology, Arsanjan Branch, Islamic Azad University, Arsanjam, Iran

${ }^{b}$ Department of Educational Science, Sepidan Branch, Islamic Azad University, Sepidan, Iran

CHRON I C L E ABSTRACT

Article history:

Received October 28, 2015

Received in revised format

November 28, 2015

Accepted February 10, 2016

Available online

February 14, 2016

Keywords:

Quality of working life

Resiliency

Social support

\begin{abstract}
In the current world, all human beings are forced to work for the living to provide their living requirements. Meeting these needs faced human with numerous psychological problems. One of the psychological problems of humanity in the current world is associated with stress caused by working conditions that human are daily forced to face with them. Finding the right solution for management of unconventional stress requires understanding the capabilities of individuals and strategies to face with them in stressful situations. This paper presents a survey to study the quality of working life and social support by considering the mediating role of resiliency. The study has accomplished among all 215 environmental guards of Mazandaran province, Iran. The results of this survey indicate that enhancing the social support of environmental guards by the relevant institutions especially communication and supporting them by friends played an important role for increasing their resilience.
\end{abstract}

\section{Introduction}

Nowadays, we need to create a positive atmosphere at the organizations, there is an increasing attention paid to the mental health of staff, and a significant number of managers believe that their organizations' effectiveness depends on the dedication and commitment of the staff.

\subsection{Quality of Working Life}

In recent years, the concept of quality of working life has been widely prevalent in organizations that promotes the growth and excellence of the activities of employees in the organization.

Quality of working life can be defined in two concepts:

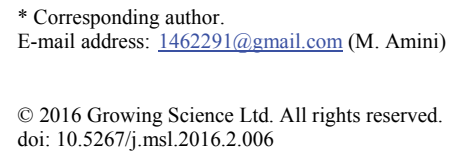


1) Objective definition: a set of real working conditions and working environment of an organization such as salaries and benefits, welfare, health and safety, participate, delegation, etc.

2) Subjective definition: specific perception of people from the quality of life; in other words, quality of working life for any individual or group of people with the same culture and attitudes, has its own characteristics.

\subsection{Social support}

Social support is a factor that causes the feelings of security and being valuable for the personnel. The researchers believe that social support prepare personnel in facing with problems. Also, existence of social support in the life of the person acts as a shield.

\subsection{The Resiliency}

Resiliency is not a passive resistance against damage or threatening situations, but resilient person is an active and constructive participant of its environment. The resilience reflects the capacity of people to stay healthy and it is resistance in harsh conditions that an individual not only overcomes difficult circumstances but also becomes stronger.

\section{Theoretical Foundations}

\subsection{Quality of Working Life}

With the complexity of the concept of quality of working life, researchers have proposed definitions to cover the theoretical failure on one side and to cover the social and economic conditions of this term, on the other side. Lawler (1982), defined the quality of working life as mental perception and understanding of the organization's employees from their physical and mental utilities of their working environment. The quality of working life, represents an organizational culture and management practices that the employees feel ownership, autonomy, responsibility and self-esteem. Quality of working life theories:
1. Kost theory
4. Mintzberg theory
7. Lawler theory
10. Walton theory
2. Morton theory
5. Stephen Robbins theory
8. Werther theory
3. Harold Kents theory
6. Cunningham and Abrel theory
9. Mills theory

\section{Indicators of quality of working life}

In a lot of programs of quality of working, there is an effort to increase the satisfaction of employees through the promotion of their intrinsic motivation. Walton's indicators (Walton, 1973) for the quality of life:

1. The fair and adequate income

2. Safe and healthy working environment

3. Providing opportunities for continuous growth and security

4. The rule of law
5. The social dependency

6. The general atmosphere of life

7. Integration

8. Development of human capabilities

\subsection{The Resiliency}

The resiliency term meanings by Oxford English Dictionary (1989a) are as follow:

1. The capacity to recover quickly from difficulties

2. The ability of a substance or object to spring back into shape

3. The ability of persons to return their original state after a period of crisis and depression 
Rutter (2012) defines resilience as a dynamic process involving positive adaptation in harsh conditions. In this definition, there are two primary requirements for resilience:

A) Exposure to a threat or really tough conditions,

B) To achieve a positive adaptation despite the attack and the threats to personal growth.

According to Tabatabai Vakili and Ghare-Aghachi (2007), factors of promoting and protecting the resiliency are divided into two factors of internal and external protection as follow:

A) Internal protective factors: including personal characteristics that facilitate and promote the resilience.

1. The use of life skills, particularly problem-solving,

6. Autonomy and independency decision-making, assertiveness and impulse control skills

2. Sociability and ability to love and be loved, the ability to 3 . Flexibility create positive relationships.

4. Humor

6. The internal locus of control

8. Optimism about the future

5. Education and training capacity

7. Spontaneity

10. Valuing their flexibility.

B) External protective factors: features in the family, school, community and peer groups that strengthen resilience:

1. Pioneer in the creation of relationships and intimate ties,

2. Valuing education, passionate interaction and less pernickety style,

3. Establishment and strengthening of clear boundaries (laws, regulations, norms),

4. Strengthening the protective and affectionate relationships with others,

5. Share responsibilities and assistance,

6. Possibility of access to resources to meet basic needs like housing, jobs, health,

7. High and realistic expectations,

8. Encouraging for target definition and dominating the situation,

9. Strengthen values ( loyalty) and life skills (such as cooperation),

10. Creating opportunities for leadership, decision-making and meaningful participation in activities,

11. Valuing the unique talents of each person.

Characteristics of resilient individuals:

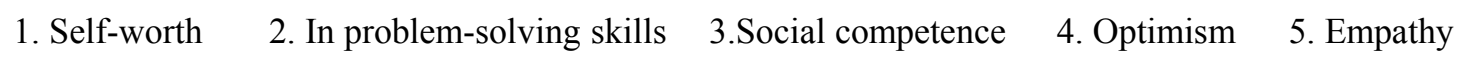

\section{Resilience models}

According to the researchers of resiliency, resiliency is by three mechanisms (Bernard, 2004a, b; Brown, 2008; Christiansen \& Evans, 2005) as follows,

A) The compensatory factor model,

B) Confront or challenge model,

C) The protection or immunity model.

The compensatory factor model, regardless of the emergence of risk, reduces the negative outputs (Christian \& Evans, 2005). Zimmerman et al. (2002) found that the compensatory factor model suggests resiliency so that the positive factors in a person's life may affect the risk to be ineffective. 
Unlike the compensatory factor model, confront model stipulates that an amount of risk is essential for psychological growth. According to Zimmerman and Aron Kumar (1994), the confront model explains a non-linear relationship between the risk and the output variable. Protection model, discuses about the resilience forces that are the foundation of resiliency. These factors are affected by culture, gender, age, socio-economic indicators (Bernard, 2004).

\subsection{Social support}

There is no doubt that, human is a social creature. It is hard to know people separated from groups linked to them. Many human behaviors, such as choice of clothing and food, talk, emotional reactions are all reflections of the social behavior and show human belonging to a particular social group (Afshari, 2007). Tardy (1985) categorized the key decisions that help to clarify the differences in the definitions of social support:

1. Distinction between the describing social support against the evaluation of customer satisfaction by providing support, because the support can be provided, but cannot be understood.

2. Knowledge from the content of social support, especially from the fact that the offered support or with suppliers is expected.

3. Investigating which network provides the available support.

Barrera (1981) and Cummins (1988) suggest two concepts of social support:

1. Receiving the support: it means having a clear emotional support and assistance, such as help from family and friends that can be measured by identifying the number of supporters and the availability of them.

2. Understanding the support: it means the availability and adequacy of different types of support. Understanding support is often known equivalent to satisfaction and social support combined with positively feelings.

\subsection{Research background}

A study in Australia on the relationship between social support and quality of working life was accomplished during pregnancy. The study was a longitudinal study in 3 hospitals in Brisbane, Australia. It examined 473 pregnant women at 36 weeks of pregnancy and 12 weeks of postpartum. To measure, the study used the quality of working life SF12 to measure the social support. The results showed that the score for the quality of working life in physical and mental was low, and social support had a significant and stable effect on the quality of life, especially after 12 weeks of maternity and mental health during the pregnancy. The relationship between social support and quality of life was independent from the parameters of education, duration for the relationship with partner, age and visits during pregnancy. Pepe (2011) reported that the level of experience, school regions, poverty level of schools, school education level and executive pay did not have a significant relationship with the resiliency of managers. However, the survey results suggested that, as there was a significant relationship between job satisfaction and resiliency, there was also a significant positive relationship between commitment and resilience. Brown (2008) in a study on 154 African-Americans investigated the relationship between social support and the resilience of the people. The results showed that a large proportion of the resilient variance was determined by the social support.

\section{Research Methodology}

In this section, the research methodology and the statistical population, sampling method and sample size were explained. Also the methods and tools for data collection, validity and reliability analyses were conducted and finally, analysis methods according to the research questions were stated. 
Type of research:

The type of study is collection and analysis of the data is based on cross-correlation.

\subsection{Hypotheses}

1. There is a significant relationship between the quality of working life and resilience.

2. There is a significant relationship between social support and resilience.

3. There is a significant relationship between social support and quality of working life.

4. Resiliency has a mediating role in the relationship between the quality of working life and social support.

\subsection{Variables}

1. The quality of work life

2. Social Protection

3. Resilience

\subsection{The population and sample size}

The population of this study included all 215 environmental guards of Mazandaran province, Iran. In order to determine the sample size Morgan table (1970) was used. According to the population size of 215 , the statistical sample size was found to be 145 . The 145 questionnaires were distributed among the people and completed. The frequency of individuals and percentage of subjects participating in the study was based on the location presented in Table 1. From 146 subjects, Mahmudabad region with the lowest frequency of 2 people $(1.4 \%)$ and Sari region with a frequency of $15(10.3 \%)$ had the lowest and highest contributions, respectively. Research data collected through questionnaire.

\section{Table 1}

The number of items and Cronbach's alpha coefficient value for the measuring instruments

\begin{tabular}{lcc}
\hline Item & Number of items & Cronbach's alpha coefficient \\
\hline Quality of working life & 24 & 0.85 \\
Social support & 23 & 0.90 \\
Resilience & 25 & 0.87 \\
\hline
\end{tabular}

\section{Data analysis}

\subsection{Descriptive statistics}

Data collection was accomplished according to information obtained from the frequency. Table 2 presents the frequency, mean, standard deviation, minimum and maximum scores of variables.

Table 2

Descriptive statistics of the data

\begin{tabular}{lccccc}
\hline Variable & Frequency & Minimum & Maximum & Average & Standard deviation \\
\hline Quality of working life & 146 & 24 & 99 & 70.08 & 13.340 \\
Social support & 146 & 23 & 81 & 67.27 & 8.593 \\
Resilience & 146 & 14 & 100 & 75.17 & 12.647 \\
\hline
\end{tabular}

To study the relationship between variables, correlation matrix was used. It is seen that, according to the correlation coefficient $(r)$ and significance level $(P)$, the relationship between all dimensions of 
three variable (quality of working life, resilience, social support and its dimensions) is significant and positive. Intensity of the relationship $(R)$ is weak between 0.1 and 0.2 , average between 0.3 and 0.6 and strong between 0.7 and 1 .

\subsection{Examining the hypotheses using linear regression technique}

Table 3 summarizes the results of regression modelling, analysis of variance and regression statistical characteristics of resilience and social support on quality of working life.

\section{Table 3}

Analysis of variance and regression statistical characteristics of resilience and social support on quality of working life

\begin{tabular}{cccccccc}
\hline Item & Sum of square & $\begin{array}{c}\text { Degree of } \\
\text { freedom }\end{array}$ & Mean square & $\mathrm{F}$ & $\mathrm{R}$ & $\mathrm{R}^{2}$ & Adjusted R \\
\hline Regression & 2552.573 & 2 & 1276.286 & & & & \\
Error & 23250.441 & 143 & 162.590 & 7.850 & 0.315 & 0.099 & 0.086 \\
Total & 25803.014 & 145 & & & & & 0.000 \\
\hline
\end{tabular}

As the results show, $F$ is significant and adjustment $R$ factor is equal to 0.086 which is equivalent to $6.8 \%$ of the variance. So the variable of quality of working life is explained by social support and resilience. Comparing the results of regression coefficients has revealed that social support with the amount of beta 0.254 had a positive effect on the quality of working life and the mediator variable of resiliency had no effect on the quality of working life.

\subsubsection{Final model of research}

The overall score for the relationship between social support in one side and the quality of work life on the other side and the intermediary role of resiliency. Fig. 1 shows this relationship.

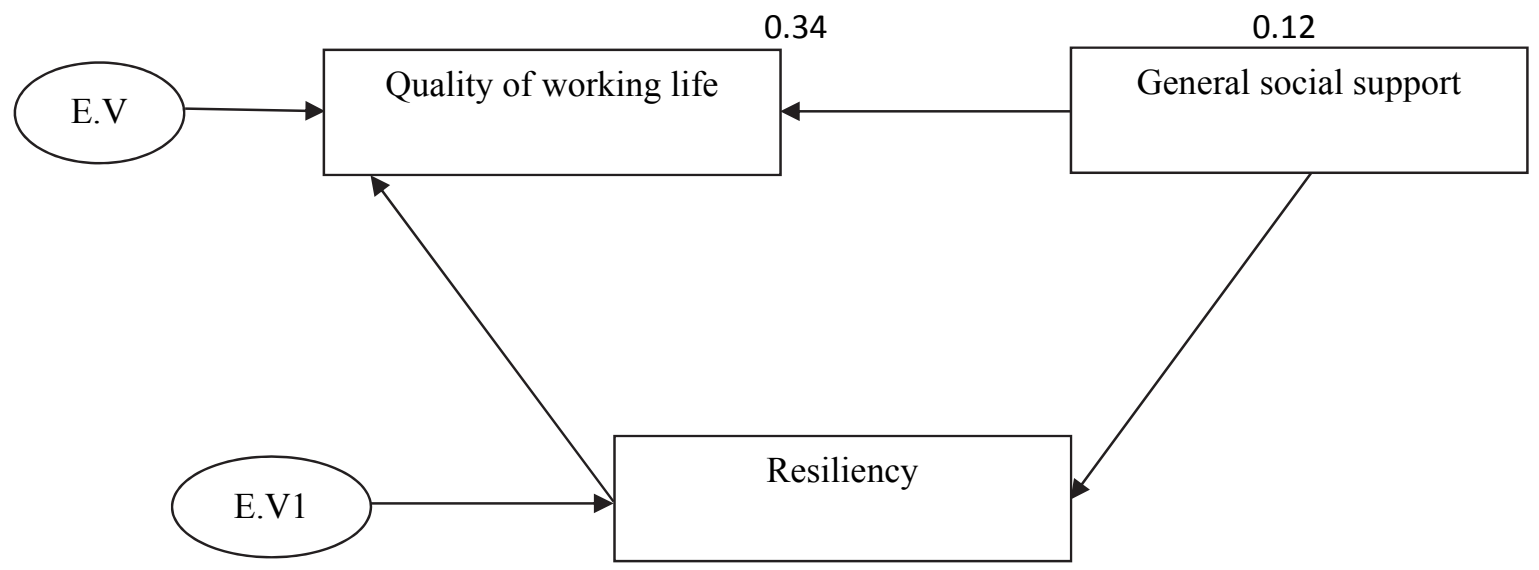

Fig. 1. The final model

In order to better clarify and examine the mediating role of resiliency, the direct and indirect effects of exogenous variables on the quality of working life are presented in Table 4.

Table 4

The direct effects, indirect effects and total effect of social protection and resiliency to the quality of working life

\begin{tabular}{clcccc}
\hline Exogenous variables & Criterion & Direct effect & Indirect effect & Total effect & Variance \\
\hline Social support & Quality of working life & 0.25 & $0.34 \times 0.12$ & 0.29 & 0.09 \\
Resiliency & Quality of working life & 0.12 & 0 & 0.12 & 0 \\
\hline The total direct and indirect effects & & & 0.41 & \\
\hline
\end{tabular}


As shown in Table 4, social support variable due to the variable of resiliency, had positive and both direct and indirect effects on the quality of working life by 0.41 and in this pattern 0.90 is equivalent to $9 \%$ of the variance between the quality of working life which is explained by the exogenous variables.

\subsection{The results to confirm or reject the hypotheses}

- First hypothesis: There is a significant relationship between the quality of working life and resilience.

- The second hypothesis: There is a significant relationship between social support and resilience.

- Third hypothesis: There is a significant relationship between social support and quality of working life.

- Fourth hypothesis: Resilience has a mediating role on the relationship between the quality of working life and social support.

\section{Conclusion}

According to research findings on the role of social support and quality of working life on resiliency of environmental guards it can be concluded that, enhancing the social support of environmental guards by the relevant institutions especially communication and supporting them by friends played an important role for increasing their resilience. So, to enhance the resilience of the people it should be planned on three levels: At the individual level, educating and encouraging people to learn various components of the solution, self-efficacy, self-esteem, etc. can enhance the resilience of people. If organizations allow their employees to be involved in some decisions, it will increase their social support and increase the satisfaction of their working life, and this partnership can be seen as promoting and protective factor of resiliency.

Experts believe that, different skills of resilience can be learned and with their education, the level of mental health, social and emotional level can be increased, which causes a feeling of satisfaction from life and quality of working. One of the factors affecting the quality of life, is self-efficacy, which affect the perceptions of skills and their ability to proper and successful functioning. The ability of social support in the organizations is known as an influential factor to face with burnout. From this study it is suggested that, training programs for stress management among the guards be implemented and resiliency skills are trained to enhance the quality of working life of environmental guards.

\section{Acknowledgement}

The authors would like to thank the anonymous referees for constructive comments on earlier version of this paper.

\section{References}

Afshari, S. (2007). The relationship between social support, self-esteem and satisfaction with life personality factors in students of Tehran. Master's thesis. Allameh Tabatabaei University, Tehran, Iran (In Persian).

Barrera, M., Jr. (1981). Social support in the adjustment of pregnant adolescents: Assessment issues. In B.H. Gottlieb (Ed.), Social networks and social support (pp. 69-96)._Beverly Hills: Sage.

Bernard, B. (2004a). Resiliency: what we have learned. San Francisco, CA: wested.

Bernard, M. E. (2004b). Emotional resilience in children: Implications for rational emotive education. Romanian Journal of Cognitive and Behavioral Psychotherapies, 4(1), 39-52.

Brown, D. L. (2008). African American resiliency: Examining racial socialization and social support as protective factors. Journal of Black Psychology, 34(1), 32-48. 
Christiansen, E. J., \& Evans, W. P. (2005). Adolescent victimization testing models of resiliency by gender. The Journal of Early Adolescence, 25(3), 298-316.

Cummins, R.C. (1988). Perceptions of social support, receipt of support behaviors, and locus of control as moderators of the effects of chronic stress. American Journal of Community Psychology, 16, 685-700.

Gillespie, B. M. (2007). The predictors of resilience in operating room nurses(Doctoral dissertation, School of Nursing and Midwifery, Faculty of Health, Griffith University).

Pepe, J. (2011). The relationship of principal resiliency to job satisfaction and work commitment: An exploratory study of K-12 public school principals in Florida (Doctoral dissertation, University of South Florida).

Lawler, E. E. (1982). Strategies for improving the quality of work life. American Psychologist, 37, 2005, 486-493.

Luthar, S. S., Cicchetti, D., \& Becker, B. (2000). The construct of resilience: A critical evaluation and guidelines for future work. Child development, 71(3), 543-562.

Rutter, M. (2012). Resilience as a dynamic concept. Development and psychopathology, 24(02), 335344.

Tabatabai Vakili, SM, Ghare-Aghachi, S. (2007). Practical Guide to collaborative school-based prevention (with an emphasis on substance abuse) Tehran: Honar-e-Avval Publication, Tehran, Iran (In Persian).

Tardy, C. H. (1985). Social support measurement. American Journal of Community Psychology, 13(2), 187-202.

Walton, R. E. (1973). Quality of working life: what is it?. Sloan Management Review, 15(1), 11.

Zimmerman, M. A., \& Arun Kumar, R. (1994). Resiliency research: Implications for schools and policy. Social Policy Report (Society for Research in child development), 8, 1-17.

Zimmerman, M. A., Bingenheimer, J. B., \& Notaro, P. C. (2002). Natural mentors and adolescent resiliency: A study with urban youth. American journal of community psychology, 30(2), 221-243.

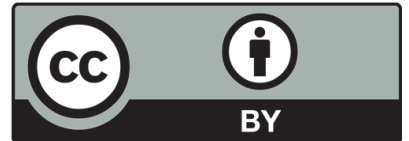

(C) 2016 by the authors; licensee Growing Science, Canada. This article is an open access article distributed under the terms and conditions of the Creative Commons Attribution (CC-BY) license (http://creativecommons.org/licenses/by/4.0/). 